\title{
Vascular Contributions to Cognitive Impairment and Treatments with Traditional Chinese Medicine
}

\author{
Xinhua Zhou, ${ }^{1}$ Guozhen Cui, ${ }^{2}$ Hisa Hui Ling Tseng, ${ }^{1}$ Simon Ming-Yuen Lee, ${ }^{1}$ \\ George Pak Heng Leung, ${ }^{3}$ Shun Wan Chan, ${ }^{3,4}$ Yiu Wa Kwan, ${ }^{5}$ and Maggie Pui Man Hoi ${ }^{1}$ \\ ${ }^{1}$ State Key Laboratory of Quality Research in Chinese Medicine and Institute of Chinese Medical Sciences, University of Macau, Macau \\ ${ }^{2}$ Department of Bioengineering, Zunyi Medical University, Zhuhai Campus, Guangdong, Zhuhai, China \\ ${ }^{3}$ State Key Laboratory of Chinese Medicine and Molecular Pharmacology, Department of Applied Biology and Chemical Technology, \\ The Hong Kong Polytechnic University, Hong Kong \\ ${ }^{4}$ Department of Food and Health Sciences, Faculty of Science and Technology, Technological and Higher Education Institute of \\ Hong Kong, Hong Kong \\ ${ }^{5}$ School of Biomedical Sciences, The Chinese University of Hong Kong, Hong Kong
}

Correspondence should be addressed to Yiu Wa Kwan; yiuwakwan@cuhk.edu.hk and Maggie Pui Man Hoi; maghoi@umac.mo

Received 31 August 2016; Revised 12 October 2016; Accepted 18 October 2016

Academic Editor: Jae Youl Cho

Copyright (C) 2016 Xinhua Zhou et al. This is an open access article distributed under the Creative Commons Attribution License, which permits unrestricted use, distribution, and reproduction in any medium, provided the original work is properly cited.

The prevalence of cognitive impairment and dementia caused by cerebrovascular disease is likely to increase with the global aging population. Vascular contributions to cognitive impairment and dementia (VCID) is a wide spectrum term used to include a diverse heterogeneous group of cognitive syndromes with vascular factors regardless of the cause of pathogenesis. VCID ranges from mild cognitive impairment to full-blown dementia with vascular dementia $(\mathrm{VaD})$ as the most severe stage. It is further complexed by the coexistence of other forms of dementia such as Alzheimer's disease (AD). Recent researches in the functions of the neurovascular unit (NVU) suggest that dysfunction of the NVU might be the cause of primary vascular events in the brain that leads to further neurodegeneration. In this review, we have briefly summarized various forms of VCID. There is currently no standard therapy for VCID or dementia. Given the fact that Traditional Chinese Medicine (TCM) has gained popularity worldwide, we also reviewed recent scientific and clinical findings on various antidementia TCM for the treatment of VCID, including Salvia miltiorrhiza, Huperzia serrata, Ligusticum chuanxiong, Ginkgo biloba, Panax ginseng, and also TCM formula Sailuotong capsule (SLT) and Fufangdanshen tablets (FFDS).

\section{Introduction}

Dementia, which describes a syndrome with a gradual decline in cognitive functioning, is a spectrum term that includes various forms of cognitive impairment especially among the elderly of our society. In 2015, the World Health Organization (WHO) estimated 47.5 million people are living with dementia worldwide, and the number is projected to be tripled to 135.5 million by 2050 [1]. The overall prevalence of dementia among people aged 60 years and above is between 5 and $10 \%$, varying among different global regions $[2,3]$. In 2010 in China, 9.19 million of people were living with various forms of dementia, and the prevalence increases quickly with age escalating from $2.6 \%$ at age $65-69$ to $60.5 \%$ at age 95-99 [4]. The most common type of dementia is Alzheimer's disease (AD), which accounts for $50-70 \%$ of all cases registered, followed by $\mathrm{VaD}$, which accounts for $25 \%$ [1, 2]. Age-related dementia is a major cause of disabilities in the elderly. Apart from the financial burdens, the social stigma associated with the loss of cognitive abilities and dependency on others also causes psychological distress in patients as well as their families. The epidemic scale of dementia poses one of the biggest challenges on global public health systems and the financial burden associated with the social care needed. The pathogenesis of dementia is complex and it involves the interactions between many different physiological systems. Traditionally, AD and VaD are classified clinically as neuropathological and cerebrovascular disorders. However, 
patients with $\mathrm{AD}$ often have mixed etiologies with both neurodegenerative and cerebrovascular pathologies $[3,5,6]$. Besides, ischemic or hemorrhagic cerebrovascular diseases or cerebral lesions caused by cardiovascular origin are commonly associated with cognitive impairments $[3,5]$. Cerebral infarctions and alterations in brain blood vessels commonly occurred in the elderly which are possibly due to age-related degeneration and other diseases $[7,8]$. Since the brain is a highly perfused organ and requires a continuous blood supply for its physiological functions, it is not surprising that damage to the cerebral circulation are associated with an increased risk of many types of dementia. In addition, epidemiology evidence indicates that $\mathrm{AD}$ and $\mathrm{VaD}$ share similar cardiovascular risk factors including apolipoprotein $\mathrm{E}$ (APOE $\varepsilon 4$ ), hypertension, hypercholesterolemia, obesity, and diabetes $[5,9,10]$, although the strength of the association between $\mathrm{AD}$ and cardiovascular risk factors could be greatly influenced by the designation of parameters, and further evaluation is needed [11, 12]. Therefore, recent researches in neurodegenerative disorders such as dementia and $\mathrm{AD}$ have focused on understanding the interplay between vascular dysfunctions and primary neurodegenerative processes. It is also suggested that cerebrovascular pathologies may be primary causes as well as contributing factors in the progression of cognitive impairment and dementia.

In this review, we have included an overview of the definitions of various forms of dementia with vascular origins, the importance of the neurovascular unit (NVU), and the preclinical and clinical investigations of using Traditional Chinese Medicine (TCM) to treat and manage cognitive decline and dementia. Management of vascular risks and symptoms is the primary approaches in treating vascular dementia, and TCM has proven ability to treat cardiovascular diseases and hypertension effectively [3,13]. Moreover, TCM formulations for the treatment of dementia-like and memory disorders have been extensively documented in the classical Chinese medical literature, including herbs such as Salvia miltiorrhiza, Huperzia serrata, Ligusticum chuanxiong, Ginkgo biloba, and Panax ginseng [14-16]. In a recent metaanalysis study, it indicated that TCM exhibited comparable efficacy and safety as Western medicine for improving the cognitive and behavior functions of patients with vascular cognitive impairment with no dementia [17]. Therefore, it is proposed that TCM has great potential uses as preventive strategies against dementia which can have positive impacts on global public health.

\section{Definitions of Vascular Dementia (VaD), Vascular Cognitive Impairment (VCI), and Alzheimer's Disease (AD)}

Dementia describes a group of syndromes relating to cognitive decline. Clinical manifestation of different forms of dementia exhibits different levels of impaired performance in various cognitive domains, including memory, learning, executive function, and behavioral changes (e.g., depressive symptoms). Cognitive impairment ranges from mild to severe declines in any cognitive domain. Alzheimer's disease
(AD) is the most common form of dementia, followed by $\mathrm{VaD}[1,2]$. In the literature, "VaD" is often used ambiguously to describe a group of clinically similar cerebrovascular disorders associated with multiple pathological features, such as multi-infarcts, single infarcts, hemorrhages, white matter hyperintensities. Moreover, the progression of the manifestation of dementia is preceded by a prodromal stage in which the patient experiences progressive cognitive decline but is still able to maintain independent daily activities [5]. This has further led to the usage of the general terms, for example, VCI or VCID, in order to include all cognitive impairments caused by cerebrovascular abnormalities, which could be quite confusing. Essentially, VCI or VCID is used to include a diverse heterogeneous group of cognitive syndromes with vascular origins regardless of the cause of pathogenesis, ranging from mild cognitive impairment to full-blown dementia, and $\mathrm{VaD}$ is the most severe stage [3, $8,18]$. The heterogeneity and complexity of VCI however make it difficult for appropriate clinical characterization. Currently there are no accepted pathological criteria for clinical diagnosis for VCI, but identifiable subtypes of VCI have been categorized. This classification system may be useful for clinicians to diagnose for the prevention and the treatment of cognitive dysfunctions. The characterization and classification of VCI are beyond the scope of the present article, and more detailed information could be found in previous reviews $[3,19,20]$. In addition, $\mathrm{AD}$ and $\mathrm{VaD}$ have traditionally been characterized as separate disorders based on the clinical diagnostic criteria. However, the "pure" form of $\mathrm{AD}$ or $\mathrm{VaD}$ is not commonly found; instead most people with dementia have mixed pathologies. Besides, $\mathrm{AD}$ frequently coexists with cerebrovascular abnormalities such as alterations in vascular structures (e.g., cerebral amyloid angiopathy, CAA) and cerebral infarctions. Accumulating evidence suggested that $\mathrm{AD}$ and $\mathrm{VaD}$ have additive effects and probably interact with each other. It has been suggested that cerebrovascular dysfunction could play a role in the development and progression of $\mathrm{AD}$. Therefore, the vascular component in the mixed pathology is particularly important for the understanding of the pathogenies of dementia. In the next section, we will give a very brief overview of the major subtypes of VCI. Note that the categorization of VCI described here does not mean to replace any existing clinical criteria for the characterization of different types of $\mathrm{VaD}$ but to offer a simplified overview for a better understanding of various VCI terminologies, especially for nonclinician researchers. The terms VCI and VCID are used interchangeable in this article.

\subsection{Major Subtypes of Vascular Contributions to Cognitive} Impairment (VCI). Many subtypes of VCI or VCID have been described before. The disorder is sometimes classified according to the location of vascular lesions, the causative vascular mechanisms, and their clinical manifestations. Chronic cerebral ischemia and arteriosclerosis were originally thought to be the cause of vascular contributed dementia but later discovered that it is cerebral infarcts rather than ischemia that causes dementia [21]. Therefore, we try to 
summarize the major subtypes of $\mathrm{VaD}$ according to the type of infarcts exhibited. Similar clinical features of VaD subtypes have been described under different names; therefore we also try to include them into specific groups. In brief, the major subtypes of VCI include vascular mild cognitive impairment (VaMCI), multi-infarct dementia (MID), strategic-infarct dementia (SID) (which is also commonly known as subcortical vascular dementia (SVD) or small vessel disease (SVD)), poststroke dementia, and mixed dementia of $\mathrm{AD}$ and $\mathrm{VaD}$. SID (or SVD) accounts for over $40 \%$ of all VCI reported $[20,21]$. Recent studies also indicated that mixed dementia particularly $\mathrm{VaD}$ in conjunction with $\mathrm{AD}$ is also commonly observed among elderly people [20, 21].

2.1.1. Vascular Mild Cognitive Impairment (VaMCI). Dementia is preceded by a stage in which mild cognitive decline is first manifested in individuals without affecting care-free daily independent activities. VaMCI is used to describe this intermediate stage between normal cognition and dementia caused by vascular diseases with the presence of cognitive impairment but it is not severe enough to fit into the criteria for $\mathrm{VaD}$ [22]. An equivalent stage occurring in $\mathrm{AD}$ is referred to as mild cognitive impairment (MCI) and is used to identify people who are at risk for the later amnestic stage of $\mathrm{AD}$. The prevalence of VaMCI is twice as much of $\mathrm{VaD}$ [22]. VaMCI is potentially treatable by management of vascular risk factors and diseases [3, 22]. Moreover, VaMCI is usually associated with other diseases such as heart failure, autoimmune disorders, and depression, and previous studies showed that cognitive functions of patients could be improved with respective treatments for these disorders with or without specific treatment for VaMCI [22-24]. VaMCI is common in stroke patients shortly after attacks, and in some patients cognition may improve as part of the stroke recovery process $[25,26]$.

2.1.2. Large-Vessels VaD: Multi-Infarct Dementia (MID). MID is caused by the "synergistic effects" caused by multiple vascular lesions ("multiple mini strokes") in the brain irrespective of specific location or volume. The mini strokes which disrupted the blood flow to the brain may occur without noticeable clinical symptoms, and over time these lead to irreversible injuries in the brain tissues. MID can be diagnosed by brain imaging techniques such as computed tomography (CT) and magnetic resonance imaging (MRI) scan. MID usually affects people between the ages of 60-75 and is more common in men than in women. However, MID is not the single most common type of $\mathrm{VaD}$ in elderly people; instead patients are more likely to have mixed dementia with both $\mathrm{AD}$ and $\mathrm{VaD}$ pathology (discussed later) [27].

\subsubsection{Small-Vessels VaD: Subcortical Ischemic Vascular De-} mentia (SIVD). SIVD is caused by the occlusion of small penetrating arteries which supply blood to the inner structures of the brain which is recognized as Binswanger's disease, lacunar infarct (LACI), or small vessel disease (SVD). SIVD is also known as silent brain infarction (SBI), and it is the most common type of $\mathrm{VaD}$ with $20-40 \%$ incidence in our community
$[28,29]$. This disease can be diagnosed using brain imaging methods, for example, CT and MRI scan. Hypertension is the major risk factor for SVID, and it is therefore potentially preventable and treatable [29]. The hereditary genetic vasculopathy Cerebral Autosomal-Dominant Arteriopathy with Subcortical Infarcts and Leukoencephalopathy (CADASIL), which causes $11 \%$ of lacunar stroke cases with leukoaraiosis in middle-aged adults, is one of the best examples of small vessel disease affecting mainly the small penetrating cerebral and leptomeningeal arteries [30].

2.1.4. Strategic-Infarct Dementia (SID). It has been traditionally recognized that single or few focal infarcts occurred in some functionally important brain regions ("strategic") such as lesions in the thalamus, caudate nucleus, lenticular nucleus, angular gyrus, and internal capsule. However, the concept of strategic infarction is under reexamination with larger prospective MRI studies to study the relationship between the extent and location of lesions and the cognitive networks [3].

2.1.5. Poststroke Dementia (PSD). Poststroke dementia (PSD), or poststroke cognitive impairment includes any type of dementia that occurs after stroke, irrespective of the leading causes, which can be vascular, neurodegenerative, or mixed. Having a stroke doubles the risk of dementia development. The prevalence of PSD in patients is about $30 \%$, which varies between age, races, diagnostic criteria, and periods after stroke [31]. The underlying causes of cognitive impairment after stroke are not known at present. It is suggested that vascular lesions caused by ischemia/hypoxia or hemorrhages, cerebral microbleeds, white matter lesions, and neurodegenerative pathologies from other conditions such as $\mathrm{AD}$ all contribute to and probably interact with each other to the pathogenesis of PSD. Increasing age is a major determinant of PSD development. It has been reported that $15 \%$ of stroke survivors at the age of 60-69 had new-onset dementia, and the prevalence was greatly increased to $26 \%$ at the ages 70 to 79 and $36 \%$ at the age over 80 years [31-34].

2.1.6. Mixed Dementia. Mixed dementia is a condition in which abnormal features of more than one type of dementia occur simultaneously in the brain, and over $44 \%$ of dementia was in fact mixed dementia with a combination of $\mathrm{AD}$ and $\mathrm{VaD}$ pathologies [35]. The coexistence of $\mathrm{AD}$ and vascular lesions is particularly common in older patients. Clinically, a spectrum of vascular diseases is related to the failure of microvasculature functions to regulate cerebral circulation and elimination of interstitial fluid and solutes in both AD and $\mathrm{VaD}$. In $\mathrm{AD}$, abnormal accumulation of amyloid-beta $(\mathrm{A} \beta)$ in the brain arterial walls, a condition known as cerebral amyloid angiopathy (CAA), leads to the weakening of the brain blood vessel wall and increases the risk of hemorrhages [36]. More recently, dementia research has focused on the importance of the neurovascular unit (NVU) at the level of cellular and molecular mechanisms (discussed later) [6, 30, 37]. Mixed dementia may also include cases of AD and 
VaD associated with any other disorders such as Parkinson's disease (PD) and dementia with Lewy bodies (DLB) [35].

The following shows general classification for cognitive impairment with vascular components.

Pathological features includes white matter hyperintensities (WMHs), multiple or single infarcts, hemorrhages, alteration of brain vessel structure, and cerebral hypoperfusion.

\section{Vascular Mild Cognitive Impairment (VaMCI)}

(i) Predementia stage: patients have cognitive decline without affecting daily independent functioning

(ii) Symptoms: amnestic, lack of attention, expressive language disorder, and visual depth perception

(iii) Diagnosis methods: brain imaging, cerebrospinal fluid tests, and so forth

The following shows severe cognitive decline.

Vascular Dementia (VaD)

Large-vessel $\mathrm{VaD}$

\section{Multi-infarct dementia (MID)}

(i) Caused by multiple infarcts (cortical and/ or subcortical) with synergistic effect irrespective of location or volume

(ii) Symptoms: getting lost, language problem, apathy, performing difficulties, emotional lability, and loss of social skills

(iii) Diagnosis methods: a neurological exam, a history of stepwise mental decline, computed tomography (CT) or magnetic resonance imaging (MRI) scans, an electroencephalogram, a transcranial Doppler, and so forth

Small vessel VaD

Subcortical ischemic vascular dementia (SIVD) (may also be known as Binswanger's disease, Lacunar infarct (LACI), silent brain infarction (SBI), small vessel disease (SVD) (e.g., CADASIL))

(i) Caused by occlusion of penetrating arteries and thus reduction of blood supply to the brain's deep structures

(ii) Symptoms: sudden hemiparesis, pseudobulbar palsy, small-stepped gait, urinary incontinence, dysarthria, dementia, and changes in effect including inappropriate laughing or crying

(iii) Diagnostic methods: assessment of cognitive deficits (criteria of clinical trial of SIVD), CT or MRI, and so forth

Strategic-infarct dementia (SID)

(i) Caused by focal infarcts in functionally important brain regions (concept of SID is under reexamination) (ii) Symptoms: mental deterioration, depression, emotional lability, apathy, cognitive, and other deficits

(iii) Diagnostic methods: MRI, CT, perfusion SPECT, and so forth

\section{Poststroke Dementia (PSD)}

(i) Including any type of dementia that occurs after stroke irrespective of causes

(ii) Symptoms: trouble with speaking and understanding, trouble with seeing in one or both eyes, cognitive impairment

(iii) Diagnostic methods: CT, PET, functional MRI, spectroscopy, and so forth

\section{Mixed Dementia}

(i) Coexistence of more than one type of dementia (e.g., VaD, Alzheimer's disease (AD), and Parkinson's disease (PD))

(ii) Symptoms: symptoms may vary, depending on the type of disease, it may be similar to those of $\mathrm{AD}$ or another specific type of dementia

(iii) Diagnostic methods: CT, MRI, and so forth

\section{Cerebral Circulation, the Neurovascular Unit (NVU), and VCI}

The pathogenesis underlying VCI has remained elusive with many complexities. However, vascular risk factors associated with the cerebral circulation such as hypertension and stroke are shared by many neurodegenerative diseases and are associated with the development of dementia [5]. The cerebral circulation is responsible for one of the most important jobs in the body to provide and regulate blood supply for the highly energy demanding central nervous system. Given the highly perfused nature of the brain, it is not surprising that any disruption or dysfunction in the cerebral circulation will lead to cognitive impairments. The brain is perfused by a sophisticated network of cerebral blood vessels. The anatomy and cellular organization of the cerebral vasculature have been described in detail previously $[38,39]$ and it is briefly introduced here.

The common carotid arteries carry blood from the heart to the brain and branch into two pairs of carotid arteries (the internal and external carotid arteries). The carotid arteries then give rise to cerebral arteries which further divide into pial arteries that run along the surface of the cortex within the pia-arachnoid. As the pial arteries penetrate into the parenchyma, they become progressively smaller, becoming intracerebral arterioles and cerebral capillaries to supply blood to the corresponding regions of the cerebral cortex. Importantly, the structures and functions of the vessels change significantly as they penetrate into the brain's inner structure. All cerebral vessels have a layer of highly specialized endothelial cells (the blood-brain barrier, BBB) that provide specific barrier functions to regulate the fluid 


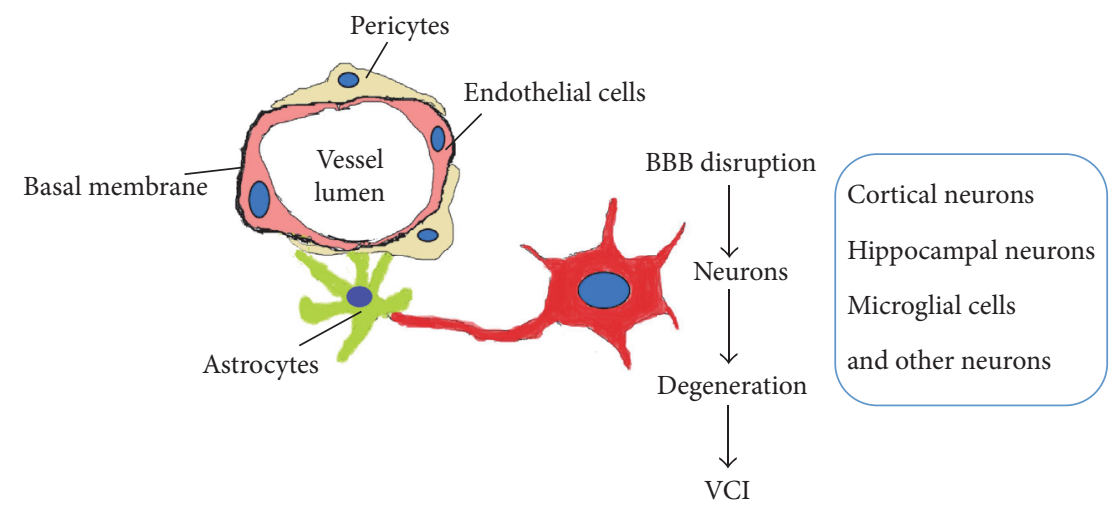

FIGURE 1: Schematic illustration of the cellular structure of the neurovascular unit (NVU). Dysfunction of the NVU causes blood-brain barrier (BBB) disruption and leads to the degeneration of neurons and VCI.

and solute exchange between the brain and the blood. The pial arteries on the surface of cerebral cortex consist of a layer of endothelial cells surrounded by 2-3 layers of smooth muscle cells and an outer layer of perivascular nerves. The smooth muscle layers are important for supporting the endothelium and regulating vessels contractility, similar to the systemic blood vessels. As the vessels penetrate in the brain and become smaller intracerebral arterioles, the smooth muscle layer becomes thinner and the vessels are completely wrapped around by astrocytic end-feet and other glia cells. The glia (including astrocytes, oligodendrocytes, and microglia) become more and more important in the maintenance and stabilities of the endothelium. As the vessels further progress and become cerebral capillaries, their endothelial cells are sealed by tight junctions, enveloped by pericytes (which replace the smooth muscle layer), and then surrounded by a continuous basement membrane (BM). End-feet of perivascular astrocytes cover the brain capillaries BM and act to integrate the communication between the endothelium and neurons.

The integrated system within a cerebral capillary that is responsible for microvascular homeostasis and neurovascular coupling (the correlation between local neuronal activity and changes in blood flow or hyperemia) is now considered a specialized functional unit known as the neurovascular unit (NVU) (Figure 1). The current understandings of the multicellular organization and functions of the NVU in normal and disease conditions are well described in recent reviews $[6,30,39]$. Essentially, the components of the NVU include all the cells present within the brain capillary (brain endothelial cells, astrocytes, pericytes, microglia, neurons, and circulating inflammatory cells) as well as the basal membrane and endothelial glycocalyx (a network of membrane-bound proteoglycans and glycoproteins covering the luminal-side of the endothelium). It is proposed that vascular risk factors such as hypertension and hypercholesterolemia damage the NVU and lead to chronic hypoperfusion, hypoxia, inflammatory activation, and oxidative stress. The formation of a hypoxic microenvironment at the NVU also directly contributes to inflammatory activation by regulating gene such as hypoxiainducible factor $1 \alpha(\mathrm{HIF} 1 \alpha)$, which serves as inducer of other genes involved in inflammatory response signaling. Tissue hypoxia and inflammatory activation in neurodegenerative diseases induce the NVU to undergo various cellular interactions and adaptations in responses, exhibiting pathophysiological features such as blood flow perturbation, vascular alterations, adaptive angiogenesis, vascular remodeling, BBB permeabilization, loss of tight junction and/or adherens junction proteins, endothelial injuries, pericytes retraction, $\mathrm{BM}$ breakdown, neurovascular uncoupling, and neuronal impairments. This highlights the association of the injury at NVU and the disruption of cerebral microcirculation as a crucial step in ischemic-hypoxic tissue damage leading to neuronal degeneration and cognitive impairment. It is suggested that dysfunction of the NVU may participate in all brain pathologies development, and the primary microvascular events may be etiological to neurological diseases, as well as being involved in diseases manifestation. Epidemiological studies have also provided strong evidence supporting that the NVU dysfunction is closely related to some neurological pathogenesis. For example, hypertension in animal models causing brain vessel rarefaction and a reduction in capillary density and microvessel formation [40]. In the hereditary CADASIL disease of small cerebral arteries, the NVU has been indicated as a predominant causative factor in the pathogenic mechanism of the disease. Structural alterations in the small penetrating cerebral and leptomeningeal arteries in CADASIL caused by pathogenic mutations in NOTCH3 (Notch homolog 3) lead to NVU impairment, causing cerebral blood flow reduction and subcortical ischemia, with lacunar infarcts correlating with cognitive decline [30]. Recently, the NVU has also been proposed to play an important part in the development of cognitive decline observed in $\operatorname{AD}[30,37,41]$. It is reported that dementia is more likely to happen when vascular lesions coexist in AD patients. Apart from cerebral amyloid angiopathy (CAA) (the accumulation of amyloid-beta $(\mathrm{A} \beta)$ in brain blood vessels), an increased concentration of soluble $\mathrm{A} \beta$ peptides in the cerebral circulation of $\mathrm{AD}$ patients is also observed [42]. The elevated level of soluble $\mathrm{A} \beta$ causes oxidative stress, and the damage on cerebral circulation induced by $\mathrm{A} \beta$ precedes cognitive decline, thus suggesting that cerebrovascular dysfunction 
might be involved in the pathogenesis of cognitive impairment [42]. All these observations emphasize the importance of the interaction between vascular and neuronal systems in the context of disease pathogenesis and imply the potential of the NVU as a target for disease prevention, treatment, and management.

\section{Therapeutic Implications for VCI with Traditional Chinese Medicine (TCM)}

Currently there is no standardized treatment regimen for VCI. Several pharmacological agents approved for AD (donepezil, galantamine, rivastigmine, and memantine, Table 1) have been tested to treat patients with $\mathrm{VaD}$ with only modest improvements on standard cognitive measures and failed to achieve regulatory approvals [3]. Other antidementia candidates for treating cerebral small vessel disease are also developed (e.g., nimodipine, huperzine) [3]. Uncertainties in diagnostic criteria and the measurements of cognitive domains further pose challenges and hurdles to the progress of drug research and development. Given the fact about the long history of TCM development and uses, many Chinese herbs have been identified for the potential treatment of the dementia-like disorders [43]. VCI belongs to the category of memory disorders according to TCM theories that is known as jian wang (健忘) (“forgetfulness") and dai bing (呆病) (“dementia”) [16, 17]. Over the last decades, many clinical trials were conducted in China to investigate TCM for treatment of cognitive impairment and dementia. Despite the enormous efforts involved, most of these trials conducted were not well-designed with limitations such as small sample size, the lack of being truly randomized, doubleblind, and placebo-controlled, and the incomplete cognitive assessments dedicated for $\mathrm{VaD}$ [44]. Thus, the genuine effectiveness of most TCM therapies assessed for treating dementia remains to be verified. Nevertheless, these studies provided a valuable resource for the search of potential antidementia drugs and therapies $[16,17,45]$. In addition, the most effective approach for VCI treatment is the management of underlying vascular risk factors and prevention of further cerebrovascular injury. Many TCM herbal ingredients have proven abilities to prevent and treat cardiovascular diseases and management of hypertension and stroke. In the following session, the experimental studies of the herbs frequently reported for the treatment of memory disorders with vascular origins are reviewed and discussed (Table 1).

4.1. Salvia miltiorrhiza. The dried root of Salvia miltiorrhiza, also known as Danshen, is a widely used TCM for the treatment and prevention of cardiovascular diseases (CVDs) such as angina pectoris, myocardial infarction, and arteriosclerosis. According to TCM theory, Danshen promotes blood flow and resolves blood stasis. Several registered pharmaceutical products containing Danshen extracts such as Danshen Dripping Pill and Fufang Danshen Tablet are widely used in clinics in the China showing effectiveness for treating CVDs [43]. The herbal extract of Danshen contains water-soluble danshensu (DSS) and salvianolic acid
TABLE 1: Current therapeutic agents and TCM for the treatment against VCI/dementia and their action targets/mechanisms.

\begin{tabular}{|c|c|}
\hline Therapeutic agents & Targets/mechanisms for VCI \\
\hline $\begin{array}{l}\text { Donepezil } \\
\text { Galantamine } \\
\text { Rivastigmine }\end{array}$ & $\begin{array}{l}\text { Acetylcholinesterase (AChE) } \\
\text { inhibition }\end{array}$ \\
\hline Memantine & $\begin{array}{l}\text { Noncompetitive NMDA receptor } \\
\text { antagonist }\end{array}$ \\
\hline Salvia miltiorrhiza & $\begin{array}{l}\text { Antiplatelet aggregation, } \\
\text { anti-inflammation, and } \\
\text { antioxidative effects }\end{array}$ \\
\hline Huperzia serrata & Selective AChE inhibition \\
\hline Ligusticum chuanxiong & $\begin{array}{l}\text { Antiapoptosis, antioxidant, } \\
\text { anti-inflammation, antiplatelet, } \\
\text { and block calcium }\left(\mathrm{Ca}^{2+}\right) \\
\text { overload }\end{array}$ \\
\hline Ginkgo biloba & $\begin{array}{l}\text { Restoring mitochondrial } \\
\text { dysfunction, improving neuronal } \\
\text { energy supplement, improving } \\
\text { compromised hippocampal } \\
\text { neurogenesis and neuroplasticity, } \\
\text { inhibiting A } \beta \text { protein } \\
\text { aggregation, decreasing blood } \\
\text { viscosity, and enhancing } \\
\text { microperfusion }\end{array}$ \\
\hline Panax ginseng & $\begin{array}{l}\text { Antioxidant, antiplatelet, } \\
\text { antihyperlipidemic, stimulation } \\
\text { of NO production, improvement } \\
\text { in blood circulation, and } \\
\text { enhancement of vasomotor tone }\end{array}$ \\
\hline Saffron & $\begin{array}{l}\text { Antioxidant and inhibiting } \\
\text { serotonin reuptake in synapses }\end{array}$ \\
\hline
\end{tabular}

B (Sal B) and lipid-soluble tanshinone I (Tan I), tanshinone IIA (Tan IIA), cryptotanshinone, and dihydrotanshinone. DSS is the major component in Danshen which possesses multiple pharmacological effects that are beneficial to the cardiovascular system, including coronary artery dilatation, antiarrhythmia, microcirculation improvement, protection of myocardial ischemia/reperfusion injury, and antiplatelet aggregation [46-48]. Danshen has been used for the prevention and treatment of cerebral infarction and the underlying mechanisms involved multiple pathways, including antihypotension, antiplatelet aggregation, and anti-inflammatory and antioxidative effects [49]. Recent animal in vivo studies have also shown that DSS exhibited protection against cerebral ischemic/reperfusion injury [50] and neuronal cytotoxicity against oxidative damage [51]. Diabetes is a vascular risk factor shared by $\mathrm{AD}$ and $\mathrm{VaD}$, and the spatial learning and memory are impaired in diabetic animals. In cognitive impaired model of streptozotocin-induced diabetic mice, DSS administration ameliorated the learning and memory deficits by attenuating advanced glycation end products(AGE-) mediated neuroinflammation [52]. The lipid-soluble Tan IIA improved learning and memory deficits in a chronic cerebral ischemia rat model in vivo by protection against free radical insults and regulating the levels of glutamate and $\gamma$-aminobutyric acid (GABA) [53]. On the other hand, 
Sal B exerted various neuroprotective and anti-inflammatory activities in vivo and in vitro [54].

4.2. Huperzia serrata. An alkaloid isolated from the Chinese herb Huperzia serrata, Huperzine A, is a natural cholinesterase inhibitor that selectively inhibits acetylcholinesterase activity and increases acetylcholine levels in brain, thereby improving cognitive functions in patients with dementia [55]. In fact, Huperzine A has been used for treating $\mathrm{AD}$ and mild memory deficits since 1994 in the China $[55,56]$. In a randomized, double-blinded placebo-controlled clinical trial with the participation of 78 patients who have mild to moderate $\mathrm{VaD}$, Huperzine A treatment significantly improved the cognitive functions in these patients [57]. Furthermore, both animal and human safety evaluations have demonstrated that Huperzine A is safe [58]. Comparing with other acetyl cholinesterase inhibitors such as galantamine, donepezil, and tacrine, Huperzine A showed a longer duration of action, better penetration of the blood-brain barrier, higher oral bioavailability, and fewer adverse reactions [59].

4.3. Ligusticum chuanxiong. Ligusticum chuanxiong is a famous medicinal herb known for its therapeutic effects for treating ischemic stroke and tetramethylpyrazine (TMP) is the major active ingredient. TMP possesses many protective cardiovascular effects, including protection of mitochondrial functions, inhibition of lipid peroxidation by free radicals, attenuating calcium $\left(\mathrm{Ca}^{2+}\right)$ overload, maintaining calcium homeostasis, inhibiting inflammatory reaction, improving endothelial cell function, and inhibiting myocardial cell apoptosis [60]. Besides, TMP inhibits angiogenesis [61], platelet aggregation, and antithrombosis and ameliorates microcirculation [47, 62]. An injectable formulation known as Guanxinning Injection (or Danshen Chuanxiong Injection) which contains extracts of Danshen and Chuanxiong in combination has displayed a high efficacy in treating hypertension and other cardiovascular diseases [63]. Apart from the beneficial cardiovascular effects, TMP is a potent neuroprotective agent. Evidence from in vivo experiments in rats with middle cerebral artery occlusion (MCAO) demonstrated that TMP has potent neuroprotective effects against ischemic brain injuries caused by transient focal cerebral ischemia reperfusion [64-67]. It has been shown that TMP effectively reduced the size of cerebral infarction and brain edema, suppressed oxidative stress and inflammatory and apoptotic responses [64, 66, 67], and could salvage neurological functions, neuronal dendritic plasticity, and behavioral disturbance in the MCAO stroke model in rats [65, 67]. In another study with scopolamine-induced memory impairment model in rat, TMP could also effectively reverse memory deficits and preserved postsynaptic protein synthesis by restoring the impaired cAMP/PKA/CREB pathways [68]. Besides, TMP attenuated iron-induced oxidative damage and apoptosis in rat cerebellar granule cells [69]. Derivatives of TMP, known as TBN and TN-2, which was synthesized with the addition of powerful free radical-scavenging nitrone moiety (Figure 2), exhibited promising neuroprotective effects.
TBN exhibited stronger antioxidative properties without affecting the thrombolytic activity of the parent TMP and produced neuroprotection against ischemic brain injuries in rat transient and permanent MCAO stroke models via the prevention of $\mathrm{Ca}^{2+}$-mediated cellular damage caused by $\mathrm{Ca}^{2+}$ influx and overload [70,71]. Furthermore, TBN protected and rescued dopaminergic neurons from 1-methyl4-phenylpyridinium- $\left(\mathrm{MPP}^{+}-\right)$and methyl-4-phenyl-1,2,3,6tetrahydropyridine- (MPTP-) or 6-hydroxyldopamine- (6OHDA-) induced damage in vitro and in vivo models of Parkinson's disease (PD) by the reduction of oxidative stress plus increased cellular antioxidative defense capacities [72]. TN-2, which has two nitrone moieties, also exhibited potent neuroprotective effects against 6-OHDA-induced neurotoxicity and MPTP/MPP ${ }^{+}$-induced dopaminergic neurons damage $[73,74]$. Another derivative of TMP, known as T006 (Figure 2), protected primary cortical neurons from neurotoxicity as well as improvement in memory deficits in APP/PS1 transgenic mice [75].

4.4. Ginkgo biloba. Ginkgo biloba is a widely cultivated tree since ancient China with the sacred belief for its healthpromoting properties. The fruits and leaves of Ginkgo biloba are used to treat various types of diseases and symptoms such as atherosclerosis, diabetes, poor circulation, fatigue, vertigo, tinnitus, and cognitive disorder [15]. A standardized extract of Ginkgo biloba (EGb 761) has been shown to exhibit potent antioxidative and antiplatelet effects and could effectively reduce damage from cerebral ischemia [76-81]. Besides, EGb 761 extract protected hippocampal neuronal and neuroglia cells against damage and enhanced the recovery of learning/memory impairments from cerebral ischemia/reperfusion in mice and rats [79-83]. Furthermore, the Ginkgo extract has been reported to improve memory deficits and cognitive dysfunctions in various experimental models of memory disorders such as chronic stress, aging, and Parkinson's disease (PD) [84-86]. Recently, EGb 761 has also been shown to enhance the functions and integrity of cerebral microvascular endothelial cells under chronic stress induced by hypoxia, hyperglycemia, or amyloid-beta $(\mathrm{A} \beta)$ protein $[87,88]$, suggesting that functions of the neurovascular unit might play an important role in the protective effects of EGb 761. Interestingly, various clinical trials have reported the efficacy of EGb 761 in improving the cognitive impairment of patients with $\mathrm{AD}$ and $\mathrm{VaD}$ [8992]. In a recent study of a 24 -week randomized controlled trial [92], once-daily preparation of EGb 761 was shown to improve cognitive functions, neuropsychiatric symptoms, and functional abilities in 333 patients with $\mathrm{AD}$ and 71 with $\mathrm{VaD}$. In the market, Ginkgo extract is developed into an herbal supplement called Gingium ${ }^{\circledR}$ which is for improving mild to moderate age-related cognitive impairments and alleviating symptoms of $\mathrm{AD}, \mathrm{VaD}$, and/or mixed dementia. It is noted that despite the encouraging evidence of the beneficial effects of Ginkgo biloba consumption in treating $\mathrm{VaD}$, the potential drug-drug interactions especially associated with long-term usage of Ginkgo extract required further evaluation. 
<smiles>Cc1nc(C)c(C)nc1C</smiles>

TMP<smiles>Cc1nc(C)c(C=[N+]([O-])C(C)(C)C)nc1C</smiles>

TBN<smiles>Cc1nc(/C=[N+](\[O-])C(C)(C)C)c(C)nc1/C=[N+](\[O-])C(C)(C)C</smiles>

$\mathrm{TN}-2$<smiles>COc1cccc(/C=N/N(C(=O)C(F)(F)F)c2ccc(C)cc2C)c1</smiles>

$\mathrm{J} 147$<smiles>Cc1ccc(N(/N=C/c2nc(C)c(C)nc2C)C(=O)C(F)(F)F)c(C)c1</smiles>

T-006

FIGURE 2: The structures of derivatives of TMP.

4.5. Panax ginseng. The root of the Panax ginseng is wellknown for many health benefits and has been widely used to promote physical strength and healthy minds. Ginseng and its active pharmacological ingredients ginsenosides have been shown to improve age-related memory and cognitive deficits; their therapeutic effects for the treatments of cerebrovascular diseases and neurodegenerative diseases have been reviewed recently [93-95]. Several studies reported that total ginseng, total saponins, and Rg1, Rb1, and Rg2 have neuroprotective effects [96-99]. Rg1 promoted the proliferation of hippocampal progenitor cells after transient global ischemia experimental brain ischemia [99], whereas Rb1 has been reported to protect hippocampal neurons against ischemia [100]. In a recent study using a VaD rat model, Rg2 improved neurological performance and memory ability of $\mathrm{VaD}$ rats after cerebral ischemia reperfusion through the modulation of antiapoptotic signaling pathways [98]. Results from the experimental AD models have also demonstrated that ginseng extract and ginsenosides are effective in protecting and alleviating neuronal damage [93]. Two clinical studies on ginseng therapy for the treatment of $\mathrm{AD}$ reported a significant improvement in cognitive performance $[101,102]$.

4.6. TCM Formulae for Treatment of VaD. In recent years, various TCM formulae for the treatment of $\mathrm{VaD}$ have been developed, for example, Sailuotong capsule (SLT) and Fufangdanshen tablets (FFDS) $[103,104]$. SLT is composed of ginseng extract (ginseng total saponins), Ginkgo biloba extract (Yinxingtong ester), and saffron extract (saffron total glycosides). The therapeutic functions of SLT are yi-qi-huoxue and hua-yu-tong-luo according to the TCM theory. Pharmacodynamics studies showed that SLT could significantly improve neurological symptoms caused by focal cerebral ischemia and improve learning and memory ability in animal models of $\mathrm{VaD}$. In healthy adults, one-week consumption of SLT improved both neurocognitive and cardiovascular functions [103]. More recently, an international multicenter phase II clinical trial of SLT in patients with mild to moderate $\mathrm{VaD}$ has been initiated in 2012 to 2014, and the clinical results/outcomes will be available soon [105]. In recent phase III clinical trial launched in 2016, which involved research teams of Xiyuan Hospital of the China Academy of Chinese Medical Sciences and the National Institute of Complementary Medicine at Western Sydney University, over 200 Australians with dementia were recruited and participated in this clinical trials, and results obtained from the pilot studies have implicated the effectiveness of SLT uses in improving learning and memory [106]. On the other hand, an ongoing doubleblind, randomized, parallel placebo-controlled clinical study on the evaluation of efficacy and safety of FFDS (Radix 
Salvia miltiorrhiza formula tablets) for patients with mild to moderate $\mathrm{VaD}$ has shown encouraging data on treating cognitive symptoms in $\mathrm{VaD}$ patients [104].

\section{Conclusions}

VCI (or VCID) is a complex form of dementia, involving both vascular and neurological aspects. The heterogeneity of VCI and the coexistence of other forms of dementia such as $\mathrm{AD}$ make it even harder for effective drug development and clinical treatment. Recently, the important roles of the neurovascular unit (NVU) in neurodegenerative diseases are rigorously studied. Unfortunately, there is no standard therapy for VCI. Most Western medicines are target-oriented whereas TCM offers therapeutic outcomes via holistic approaches which are probably appropriate for treating VCI which involved various factors. Evidence-based TCM therapies have shown some promising results for treating the symptoms of cognitive impairments. Therefore, TCM serves as a rich resource for therapeutic developments for treating dementia, and it will be of interest to further investigate the actions of potential TCM ingredients on the functions of NVU.

\section{Competing Interests}

The authors declare that they have no competing interests.

\section{Acknowledgments}

This work was supported by Research Committee of University of Macau (MYRG2015-00161-ICMS-QRCM), Macau Science and Technology Development Fund (FDCT/127/ 2014/A3), and National Natural Science Foundation of China (NSFC81403139).

\section{References}

[1] World Health Organization (WHO), "Dementia," Fact Sheet 362, WHO, 2015.

[2] Alzheimer's Disease International, World Alzheimer's Report 2015: Global Impact of Dementia, 2015.

[3] P. B. Gorelick, A. Scuteri, S. E. Black et al., "Vascular contributions to cognitive impairment and dementia: a statement for healthcare professionals from the American Heart Association/American Stroke Association," Stroke, vol. 42, no. 9, pp. 2672-2713, 2011.

[4] K. Y. Chan, W. Wang, J. J. Wu et al., "Epidemiology of Alzheimer's disease and other forms of dementia in China, 1990-2010: a systematic review and analysis," The Lancet, vol. 381, no. 9882, pp. 2016-2023, 2013.

[5] M. Wiesmann, A. J. Kiliaan, and J. A. Claassen, "Vascular aspects of cognitive impairment and dementia," Journal of Cerebral Blood Flow and Metabolism, vol. 33, no. 11, pp. 16961706, 2013.

[6] B. V. Zlokovic, "Neurovascular pathways to neurodegeneration in Alzheimer's disease and other disorders," Nature Reviews Neuroscience, vol. 12, no. 12, pp. 723-738, 2011.

[7] J. E. Simpson, S. B. Wharton, J. Cooper et al., "Alterations of the blood-brain barrier in cerebral white matter lesions in the ageing brain," Neuroscience Letters, vol. 486, no. 3, pp. 246-251, 2010.

[8] A. D. Korczyn, V. Vakhapova, and L. T. Grinberg, "Vascular dementia," Journal of the Neurological Sciences, vol. 322, no. 12, pp. 2-10, 2012.

[9] N. Lorius, J. J. Locascio, D. M. Rentz et al., "Vascular disease and risk factors are associated with cognitive decline in the Alzheimer disease spectrum," Alzheimer Disease and Associated Disorders, vol. 29, no. 1, pp. 18-25, 2015.

[10] B. N. Dugger, M. Malek-Ahmadi, S. E. Monsell et al., "A cross-sectional analysis of late-life cardiovascular factors and their relation to clinically defined neurodegenerative diseases," Alzheimer Disease \& Associated Disorders, vol. 30, no. 3, pp. 223-229, 2016.

[11] M. C. Power, J. Weuve, J. J. Gagne, M. B. McQueen, A. Viswanathan, and D. Blackera, "The association between blood pressure and incident Alzheimer disease: a systematic review and meta-analysis," Epidemiology, vol. 22, no. 5, pp. 646-659, 2011.

[12] C. Purnell, S. Gao, C. M. Callahan, and H. C. Hendrie, "Cardiovascular risk factors and incident alzheimer disease: a systematic review of the literature," Alzheimer Disease and Associated Disorders, vol. 23, no. 1, pp. 1-10, 2009.

[13] P.-P. Hao, F. Jiang, Y.-G. Chen et al., "Traditional Chinese medication for cardiovascular disease," Nature Reviews Cardiology, vol. 12, no. 2, pp. 115-122, 2015.

[14] Y.-S. Ho, K.-F. So, and R. C.-C. Chang, "Drug discovery from Chinese medicine against neurodegeneration in Alzheimer's and vascular dementia," Chinese Medicine, vol. 6, article 15, 2011.

[15] Z.-K. Sun, H.-Q. Yang, and S.-D. Chen, "Traditional Chinese medicine: a promising candidate for the treatment of Alzheimer's disease," Translational Neurodegeneration, vol. 2, no. 1, article 6, 2013.

[16] B. H. May, C. Lu, Y. Lu, A. L. Zhang, and C. C. L. Xue, "Chinese herbs for memory disorders: a review and systematic analysis of classical herbal literature," Journal of Acupuncture and Meridian Studies, vol. 6, no. 1, pp. 2-11, 2013.

[17] M. Feng, J. Lu, B. H. May et al., "Chinese herbal medicine for patients with vascular cognitive impairment no dementia: protocol for a systematic review," BMJ Open, vol. 6, no. 3, Article ID e010295, 2016.

[18] R. A. Corriveau, F. Bosetti, M. Emr et al., "The Science of Vascular Contributions to Cognitive Impairment and Dementia (VCID): a framework for advancing research priorities in the cerebrovascular biology of cognitive decline," Cellular and Molecular Neurobiology, vol. 36, no. 2, pp. 281-288, 2016.

[19] J. Ma, Y. Zhang, and Q. Guo, "Comparison of vascular cognitive impairment_no dementia by multiple classification methods," International Journal of Neuroscience, vol. 125, no. 11, pp. 823830, 2015.

[20] J. V. Bowler, "Vascular cognitive impairment," Journal of Neurology, Neurosurgery, and Psychiatry, vol. 76, supplement 5, pp. v35-v44, 2005.

[21] J. V. Bowler, "Modern concept of vascular cognitive impairment," British Medical Bulletin, vol. 83, no. 1, pp. 291-305, 2007.

[22] A. Consoli, M. Pasi, and L. Pantoni, "Vascular mild cognitive impairment: concept, definition, and directions for future studies," Aging Clinical and Experimental Research, vol. 24, no. 2, pp. 113-116, 2012.

[23] J. G. Hanly, N. M. Walsh, J. D. Fisk et al., "Cognitive impairment and autoantlbodies in systemic lupus erythematosus," Rheumatology, vol. 32, no. 4, pp. 291-296, 1993. 
[24] M. Petri, M. Naqibuddin, K. A. Carson et al., "Depression and cognitive impairment in newly diagnosed systemic lupus erythematosus," Journal of Rheumatology, vol. 37, no. 10, pp. 2032-2038, 2010.

[25] P. S. Sachdev, X. Chen, H. Brodaty, C. Thompson, A. Altendorf, and W. Wen, "The determinants and longitudinal course of post-stroke mild cognitive impairment," Journal of the International Neuropsychological Society, vol. 15, no. 6, pp. 915-923, 2009.

[26] D. W. Desmond, J. T. Moroney, M. Sano, and Y. Stern, "Recovery of cognitive function after stroke," Stroke, vol. 27, no. 10, pp. 1798-1803, 1996.

[27] Neuropathology Group of the Medical Research Council Cognitive Function and Ageing Study (MRC CFAS), "Pathological correlates of late-onset dementia in a multicentre, communitybased population in England and Wales," The Lancet, vol. 357, no. 9251, pp. 169-175, 2001.

[28] S. E. Vermeer, W. T. Longstreth Jr., and P. J. Koudstaal, "Silent brain infarcts: a systematic review," The Lancet Neurology, vol. 6, no. 7, pp. 611-619, 2007.

[29] H. C. Chui, "Subcortical ischemic vascular dementia," Neurologic Clinics, vol. 25, no. 3, pp. 717-740, 2007.

[30] D. B. Stanimirovic and A. Friedman, "Pathophysiology of the neurovascular unit: disease cause or consequence," Journal of Cerebral Blood Flow and Metabolism, vol. 32, no. 7, pp. 12071221, 2012.

[31] D. Leys, H. Hénon, M.-A. Mackowiak-Cordoliani, and F. Pasquier, "Poststroke dementia," The Lancet Neurology, vol. 4, no. 11, pp. 752-759, 2005.

[32] D. Inzitari, A. Di Carlo, G. Pracucci et al., "Incidence and determinants of poststroke dementia as defined by an informant interview method in a hospital-based stroke registry," Stroke, vol. 29, no. 10, pp. 2087-2093, 1998.

[33] J.-H. Sun, L. Tan, and J.-T. Yu, "Post-stroke cognitive impairment: epidemiology, mechanisms and management," Annals of Translational Medicine, vol. 2, no. 8, article 80, 2014.

[34] T. Pohjasvaara, T. Erkinjuntti, R. Ylikoski, M. Hietanen, R. Vataja, and M. Kaste, "Clinical determinants of poststroke dementia," Stroke, vol. 29, no. 1, pp. 75-81, 1998.

[35] K. M. Langa, N. L. Foster, and E. B. Larson, "Mixed dementia: emerging concepts and therapeutic implications," Journal of the American Medical Association, vol. 292, no. 23, pp. 2901-2908, 2004.

[36] R. O. Weller, D. Boche, and J. A. R. Nicoll, "Microvasculature changes and cerebral amyloid angiopathy in Alzheimer's disease and their potential impact on therapy," Acta Neuropathologica, vol. 118, no. 1, pp. 87-102, 2009.

[37] C. Iadecola, "Neurovascular regulation in the normal brain and in Alzheimer's disease," Nature Reviews Neuroscience, vol. 5, no. 5, pp. 347-360, 2004.

[38] M. J. Cipolla, The Cerebral Circulation, Morgan \& Claypool Life Sciences, San Rafael, Calif, USA, 2009.

[39] E. A. Neuwelt, B. Bauer, C. Fahlke et al., "Engaging neuroscience to advance translational research in brain barrier biology," Nature Reviews Neuroscience, vol. 12, no. 3, pp. 169-182, 2011.

[40] P. W. Pires, C. M. Dams Ramos, N. Matin, and A. M. Dorrance, "The effects of hypertension on the cerebral circulation," American Journal of Physiology-Heart and Circulatory Physiology, vol. 304, no. 12, pp. H1598-H1614, 2013.

[41] C. Iadecola, "The overlap between neurodegenerative and vascular factors in the pathogenesis of dementia," Acta Neuropathologica, vol. 120, no. 3, pp. 287-296, 2010.
[42] Z. S. Katusic and S. A. Austin, "Endothelial nitric oxide: protector of a healthy mind," European Heart Journal, vol. 35, no. 14, pp. 888-894, 2014.

[43] Z. Lin, J. Gu, J. Xiu, T. Mi, J. Dong, and J. K. Tiwari, “Traditional Chinese medicine for senile dementia," Evidence-Based Complementary and Alternative Medicine, vol. 2012, Article ID 692621, 13 pages, 2012.

[44] X. Qin, Y. Liu, Y. Wu et al., "A meta-analysis of Chinese herbal medicines for vascular dementia," Neural Regeneration Research, vol. 8, no. 18, pp. 1685-1692, 2013.

[45] J. Liang, F. Li, C. Wei et al., "Rationale and design of a multicenter, phase 2 clinical trial to investigate the efficacy of traditional Chinese medicine SaiLuoTong in vascular dementia," Journal of Stroke \& Cerebrovascular Diseases, vol. 23, no. 10, pp. 26262634, 2014.

[46] G. Cui, L. Shan, M. Hung et al., "A novel Danshensu derivative confers cardioprotection via PI3K/Akt and Nrf2 pathways," International Journal of Cardiology, vol. 168, no. 2, pp. 13491359, 2013.

[47] G. Cui, L. Shan, L. Guo et al., "Novel anti-thrombotic agent for modulation of protein disulfide isomerase family member ERp57 for prophylactic therapy," Scientific Reports, vol. 5, article 10353, 2015.

[48] G. Z. Cui, L. C. Shan, I. K. Chu et al., "Identification of disulfide isomerase ERp57 as a target for small molecule cardioprotective agents," RSC Advances, vol. 5, no. 91, pp. 74605-74610, 2015.

[49] T.-H. Lin and C.-L. Hsieh, "Pharmacological effects of Salvia miltiorrhiza (Danshen) on cerebral infarction," Chinese Medicine, vol. 5, article 22, 2010.

[50] C. Guo, Y. Yin, J. Duan et al., "Neuroprotective effect and underlying mechanism of sodium danshensu [3-(3,4dihydroxyphenyl) lactic acid from Radix and Rhizoma Salviae miltiorrhizae $=$ Danshen] against cerebral ischemia and reperfusion injury in rats," Phytomedicine, vol. 22, no. 2, pp. 283-289, 2015.

[51] C.-M. Chong, Z.-Y. Zhou, V. Razmovski-Naumovski et al., "Danshensu protects against 6-hydroxydopamine-induced damage of PC12 cells in vitro and dopaminergic neurons in zebrafish," Neuroscience Letters, vol. 543, pp. 121-125, 2013.

[52] T. Wang, F. Fu, B. Han, L. Zhang, and X. Zhang, "Danshensu ameliorates the cognitive decline in streptozotocin-induced diabetic mice by attenuating advanced glycation end productmediated neuroinflammation," Journal of Neuroimmunology, vol. 245, no. 1-2, pp. 79-86, 2012.

[53] Z. He, Z. Pan, and W. Lu, "Neuroprotective effects of tanshinone IIA on vascular dementia in rats," Zhongguo Zhongyao Zazhi, vol. 35, no. 14, pp. 1883-1886, 2010.

[54] M. Imanshahidi and H. Hosseinzadeh, "The pharmacological effects of Salvia species on the central nervous system," Phytotherapy Research, vol. 20, no. 6, pp. 427-437, 2006.

[55] S.-H. Xing, C.-X. Zhu, R. Zhang, and L. An, "Huperzine a in the treatment of Alzheimer's disease and vascular dementia: a meta-analysis," Evidence-Based Complementary and Alternative Medicine, vol. 2014, Article ID 363985, 10 pages, 2014.

[56] Z. Hao, M. Liu, Z. Liu, and D. Lv, "Huperzine A for vascular dementia," Cochrane Database of Systematic Reviews, no. 2, Article ID CD007365, 2009.

[57] Z. Q. Xu, X. M. Liang, W. Juan, Y. F. Zhang, C. X. Zhu, and X. J. Jiang, "Treatment with Huperzine A improves cognition in vascular dementia patients," Cell Biochemistry and Biophysics, vol. 62, no. 1, pp. 55-58, 2012. 
[58] A. Zangara, "The psychopharmacology of huperzine A: an alkaloid with cognitive enhancing and neuroprotective properties of interest in the treatment of Alzheimer's disease," Pharmacology Biochemistry and Behavior, vol. 75, no. 3, pp. 675-686, 2003.

[59] G. T. Ha, R. K. Wong, and Y. Zhang, "Huperzine a as potential treatment of Alzheimer's disease: an assessment on chemistry, pharmacology, and clinical studies," Chemistry and Biodiversity, vol. 8, no. 7, pp. 1189-1204, 2011.

[60] W. Qian, X. Xiong, Z. Fang, H. Lu, and Z. Wang, "Protective effect of tetramethylpyrazine on myocardial ischemiareperfusion injury," Evidence-Based Complementary and Alternative Medicine, vol. 2014, Article ID 107501, 9 pages, 2014.

[61] X. Cai, Z. Chen, X. Pan et al., "Inhibition of angiogenesis, fibrosis and thrombosis by tetramethylpyrazine: mechanisms contributing to the SDF-1/CXCR4 axis," PLoS ONE, vol. 9, no. 2, Article ID e88176, 2014.

[62] P. Wang, C.-H. Luo, Q.-X. Wang, Q.-Y. Li, P. Li, and X.-H. Yuan, "Antithrombotic effect of ligustrazine hydrochloride injection on the model of induced arteriovenous shunt thrombosis," Journal of Research in Medical Sciences, vol. 18, no. 8, pp. 704706, 2013.

[63] X. Wang and M. Zhao, "Ligustrazine and Salvia miltiorrhiza injection solution in complementary therapy of pregnancyinduced hypertension: clinical analysis of 60 cases," Academic Journal of the First Medical College of PLA, vol. 23, no. 9, pp. 969-971, 2003.

[64] S.-L. Liao, T.-K. Kao, W.-Y. Chen et al., "Tetramethylpyrazine reduces ischemic brain injury in rats," Neuroscience Letters, vol. 372, no. 1-2, pp. 40-45, 2004.

[65] J.-B. Lin, C.-J. Zheng, X. Zhang, J. Chen, W.-J. Liao, and Q. Wan, "Effects of tetramethylpyrazine on functional recovery and neuronal dendritic plasticity after experimental stroke," Evidence-Based Complementary and Alternative Medicine, vol. 2015, Article ID 394926, 10 pages, 2015.

[66] Y. Chang, G. Hsiao, S.-H. Chen et al., "Tetramethylpyrazine suppresses HIF- $1 \alpha$, TNF- $\alpha$, and activated caspase- 3 expression in middle cerebral artery occlusion-induced brain ischemia in rats," Acta Pharmacologica Sinica, vol. 28, no. 3, pp. 327-333, 2007.

[67] T.-K. Kao, Y.-C. Ou, J.-S. Kuo et al., "Neuroprotection by tetramethylpyrazine against ischemic brain injury in rats," Neurochemistry International, vol. 48, no. 3, pp. 166-176, 2006.

[68] W. Wu, X. Yu, X.-P. Luo, S.-H. Yang, and D. Zheng, “Tetramethylpyrazine protects against scopolamine-induced memory impairments in rats by reversing the cAMP/PKA/CREB pathway," Behavioural Brain Research, vol. 253, pp. 212-216, 2013.

[69] Z. Zhang, T. Wei, J. Hou, G. Li, S. Yu, and W. Xin, "Iron-induced oxidative damage and apoptosis in cerebellar granule cells: attenuation by tetramethylpyrazine and ferulic acid," European Journal of Pharmacology, vol. 467, no. 1-3, pp. 41-47, 2003.

[70] Y. Sun, J. Jiang, Z. Zhang et al., "Antioxidative and thrombolytic TMP nitrone for treatment of ischemic stroke," Bioorganic and Medicinal Chemistry, vol. 16, no. 19, pp. 8868-8874, 2008.

[71] Y. Sun, P. Yu, G. Zhang et al., "Therapeutic effects of tetramethylpyrazine nitrone in rat ischemic stroke models," Journal of Neuroscience Research, vol. 90, no. 8, pp. 1662-1669, 2012.

[72] B. Guo, D. Xu, H. Duan et al., "Therapeutic effects of multifunctional tetramethylpyrazine nitrone on models of Parkinson's disease in vitro and in vivo," Biological and Pharmaceutical Bulletin, vol. 37, no. 2, pp. 274-285, 2014.

[73] D. Xu, H. Duan, Z. Zhang et al., "The novel tetramethylpyrazine bis-nitrone $(\mathrm{TN}-2)$ protects against $\mathrm{MPTP} / \mathrm{MPP}+$-induced neurotoxicity via inhibition of mitochondrial-dependent apoptosis," Journal of Neuroimmune Pharmacology, vol. 9, no. 2, pp. 245-258, 2014.

[74] D.-P. Xu, K. Zhang, Z.-J. Zhang et al., "A novel tetramethylpyrazine bis-nitrone (TN-2) protects against 6-hydroxyldopamine-induced neurotoxicity via modulation of the NF- $\kappa \mathrm{B}$ and the PKC $\alpha / \mathrm{PI} 3-\mathrm{K} /$ Akt pathways," Neurochemistry International, vol. 78, pp. 76-85, 2014.

[75] H.-Y. Chen, D.-P. Xu, G.-L. Tan et al., "A potent multi-functional neuroprotective derivative of tetramethylpyrazine," Journal of Molecular Neuroscience, vol. 56, no. 4, pp. 977-987, 2015.

[76] R. Bridi, F. P. Crossetti, V. M. Steffen, and A. T. Henriques, “The antioxidant activity of standardized extract of Ginkgo biloba (EGb 761) in rats," Phytotherapy Research, vol. 15, no. 5, pp. 449451, 2001.

[77] J. M. Kim, S. H. Ryou, Y. H. Kang, and J. S. Kang, "Effect of Ginkgo biloba leaf powder and extract on plasma and liver lipids, platelet aggregation and erythrocyte $\mathrm{Na}+$ efflux in rats fed hypercholesterolemic diet," The FASEB Journal, vol. 25, no. 1, supplement, pp. 980-988, 2011.

[78] E. J. Lee, H.-Y. Chen, T.-S. Wu, T.-Y. Chen, I. A. Ayoub, and K. I. Maynard, "Acute administration of Ginkgo biloba extract (EGb 761) affords neuroprotection against permanent and transient focal cerebral ischemia in Sprague-Dawley rats," Journal of Neuroscience Research, vol. 68, no. 5, pp. 636-645, 2002.

[79] K. Chandrasekaran, Z. Mehrabian, B. Spinnewyn, C. Chinopoulos, K. Drieu, and G. Fiskum, "Neuroprotective effects of bilobalide, a component of Ginkgo biloba extract (EGb 761) in global brain ischemia and in excitotoxicityinduced neuronal death," Pharmacopsychiatry, vol. 36, supplement 1, pp. S89-S94, 2003.

[80] I. Domoráková, J. Burda, E. Mechírová, and M. Feriková, "Mapping of rat hippocampal neurons with NeuN after ischemia/reperfusion and Ginkgo biloba extract (EGb 761) pretreatment," Cellular and Molecular Neurobiology, vol. 26, no. 7-8, pp. 1193-1204, 2006.

[81] R. A. Paganelli, A. Benetoli, and H. Milani, "Sustained neuroprotection and facilitation of behavioral recovery by the Ginkgo biloba extract, EGb 761, after transient forebrain ischemia in rats," Behavioural Brain Research, vol. 174, no. 1, pp. 70-77, 2006.

[82] M. Jahanshahi, E. Nikmahzar, N. Yadollahi, and K. Ramazani, "Protective effects of Ginkgo biloba extract (EGB 761) on astrocytes of rat hippocampus after exposure with scopolamine," Anatomy \& Cell Biology, vol. 45, no. 2, pp. 92-96, 2012.

[83] M. Jahanshahi, E. G. Nickmahzar, and F. Babakordi, “The effect of Ginkgo biloba extract on scopolamine-induced apoptosis in the hippocampus of rats," Anatomical Science International, vol. 88 , no. 4, pp. 217-222, 2013.

[84] K. Takuma, Y. Hoshina, S. Arai et al., "Ginkgo biloba extract EGb 761 attenuates hippocampal neuronal loss and cognitive dysfunction resulting from chronic restraint stress in ovariectomized rats," Neuroscience, vol. 149, no. 2, pp. 256-262, 2007.

[85] K. Tanaka, R. F. Galduroz, L. T. Gobbi, and J. C. Galduroz, "Ginkgo biloba extract in an animal model of parkinson's disease: a systematic review," Current Neuropharmacology, vol. 11, no. 4, pp. 430-435, 2013.

[86] M. L. Ribeiro, L. M. Moreira, D. P. Arçari et al., "Protective effects of chronic treatment with a standardized extract of Ginkgo biloba L. in the prefrontal cortex and dorsal hippocampus of middle-aged rats," Behavioural Brain Research, vol. 313, pp. 144-150, 2016. 
[87] F.-L. Yan, Y. Zheng, and F.-D. Zhao, "Effects of ginkgo biloba extract EGb761 on expression of RAGE and LRP-1 in cerebral microvascular endothelial cells under chronic hypoxia and hypoglycemia," Acta Neuropathologica, vol. 116, no. 5, pp. 529$535,2008$.

[88] W.-B. Wan, L. Cao, L.-M. Liu et al., "EGb761 provides a protective effect against A $\beta 1-42$ oligomer-induced cell damage and blood-brain barrier disruption in an in vitro bEnd. 3 endothelial model," PLoS ONE, vol. 9, no. 11, Article ID 0113126, 2014.

[89] S. Kanowski, W. M. Herrmann, K. Stephan, W. Wierich, and R. Hörr, "Proof of efficacy of the ginkgo biloba special extract EGb 761 in outpatients suffering from mild to moderate primary degenerative dementia of the Alzheimer type or multi-infarct dementia," Pharmacopsychiatry, vol. 29, no. 2, pp. 47-56, 1996.

[90] J. A. Mix and W. D. Crews Jr., "A double-blind, placebocontrolled, randomized trial of Ginkgo biloba extract EGb 761 ${ }^{\circledR}$ in a sample of cognitively intact older adults: neuropsychological findings," Human Psychopharmacology, vol. 17, no. 6, pp. 267-277, 2002.

[91] O. Napryeyenko, G. Sonnik, and I. Tartakovsky, "Efficacy and tolerability of Ginkgo bilobaextract EGb 761 by type of dementia: analyses of a randomised controlled trial," Journal of the Neurological Sciences, vol. 283, no. 1-2, pp. 224-229, 2009.

[92] R. Ihl, M. Tribanek, and N. Bachinskaya, "Efficacy and tolerability of a once daily formulation of Ginkgo biloba extract EGb $761^{\circledR}$ in Alzheimer's disease and vascular dementia: results from a randomised controlled trial," Pharmacopsychiatry, vol. 45, no. 2, pp. 41-46, 2012.

[93] I.-H. Cho, "Efects of Panax ginseng in neurodegenerative diseases," Journal of Ginseng Research, vol. 36, no. 4, pp. 342353, 2012.

[94] V. Rastogi, J. Santiago-Moreno, and S. Doré, "Ginseng: a promising neuroprotective strategy in stroke," Frontiers in Cellular Neuroscience, vol. 8, article 457, 2015.

[95] S. F. Nabavi, A. Sureda, S. Habtemariam, and S. M. Nabavi, "Ginsenoside Rd and ischemic stroke; a short review of literatures," Journal of Ginseng Research, vol. 39, no. 4, pp. 299-303, 2015.

[96] T.-F. Lee, Y.-J. Shiao, C.-F. Chen, and L. C. H. Wang, "Effect of ginseng saponins on $\beta$-amyloid-suppressed acetylcholine release from rat hippocampal slices," Planta Medica, vol. 67, no. 7, pp. 634-637, 2001.

[97] H. Zhao, Q. Li, X. Pei et al., "Long-term ginsenoside administration prevents memory impairment in aged C57BL/6J mice by up-regulating the synaptic plasticity-related proteins in hippocampus," Behavioural Brain Research, vol. 201, no. 2, pp. 311-317, 2009.

[98] G. Zhang, A. Liu, Y. Zhou, X. San, T. Jin, and Y. Jin, "Panax ginseng ginsenoside- $\mathrm{Rg} 2$ protects memory impairment via anti-apoptosis in a rat model with vascular dementia," Journal of Ethnopharmacology, vol. 115, no. 3, pp. 441-448, 2007.

[99] L. Shen and J. Zhang, "NMDA receptor and iNOS are involved in the effects of ginsenoside Rg1 on hippocampal neurogenesis in ischemic gerbils," Neurological Research, vol. 29, no. 3, pp. 270-273, 2007.

[100] J.-H. Lim, T.-C. Wen, S. Matsuda et al., "Protection of ischemic hippocampal neurons by ginsenoside Rbl, a main ingredient of ginseng root," Neuroscience Research, vol. 28, no. 3, pp. 191-200, 1997.

[101] J.-H. Heo, S.-T. Lee, K. Chu et al., "An open-label trial of Korean red ginseng as an adjuvant treatment for cognitive impairment in patients with Alzheimer's disease," European Journal of Neurology, vol. 15, no. 8, pp. 865-868, 2008.

[102] S.-T. Lee, K. Chu, J.-Y. Sim, J.-H. Heo, and M. Kim, "Panax ginseng enhances cognitive performance in Alzheimer disease," Alzheimer Disease and Associated Disorders, vol. 22, no. 3, pp. 222-226, 2008.

[103] G. Z. Steiner, A. Yeung, J.-X. Liu et al., "The effect of Sailuotong (SLT) on neurocognitive and cardiovascular function in healthy adults: a randomised, double-blind, placebo controlled crossover pilot trial," BMC Complementary and Alternative Medicine, vol. 16, article 15, 2016.

[104] J. Tian, J. Shi, M. Wei et al., "The efficacy and safety of Fufangdanshen tablets (Radix Salviae miltiorrhizae formula tablets) for mild to moderate vascular dementia: a study protocol for a randomized controlled trial," Trials, vol. 17, no. 1, p. 281, 2016.

[105] J. Liang, F. Li, C. Wei et al., "Rationale and design of a multicenter, phase 2 clinical trial to investigate the efficacy of traditional Chinese medicine sailuotong in vascular dementia," Journal of Stroke and Cerebrovascular Diseases, vol. 23, no. 10, pp. 2626-2634, 2014.

[106] Chinese herbs may be key to unlocking dementia, November 2015, http://nicm.edu.au/news/chinese_herbs_may_be_key_to_ unlocking_dementia. 


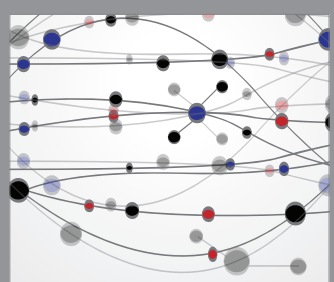

The Scientific World Journal
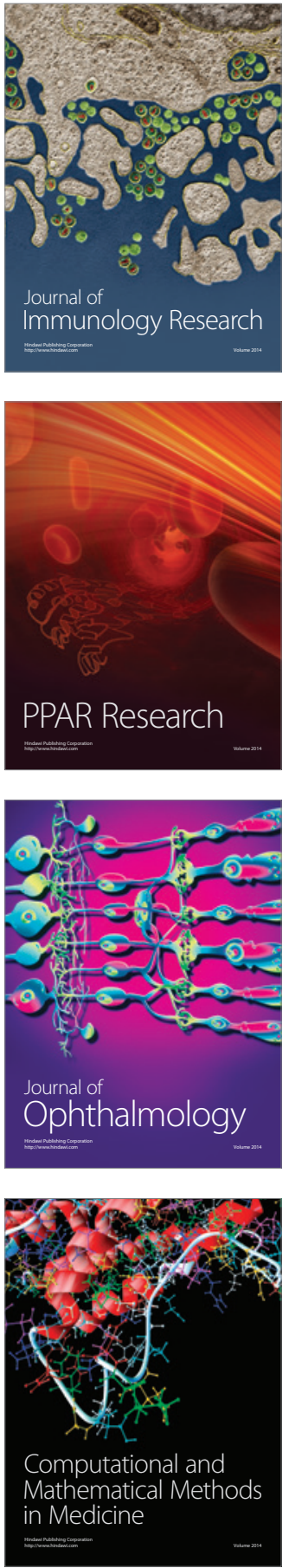

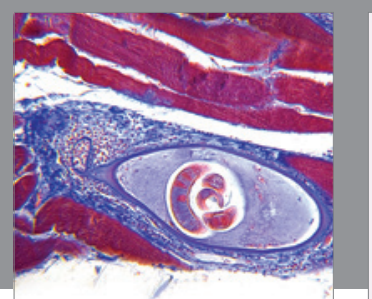

Gastroenterology Research and Practice

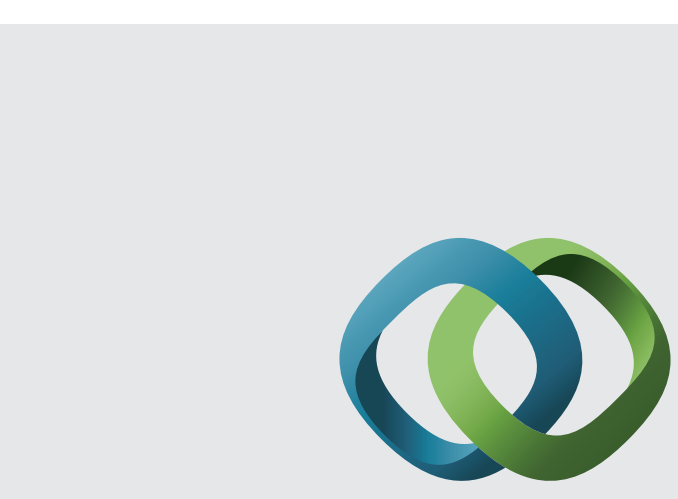

\section{Hindawi}

Submit your manuscripts at

http://www.hindawi.com
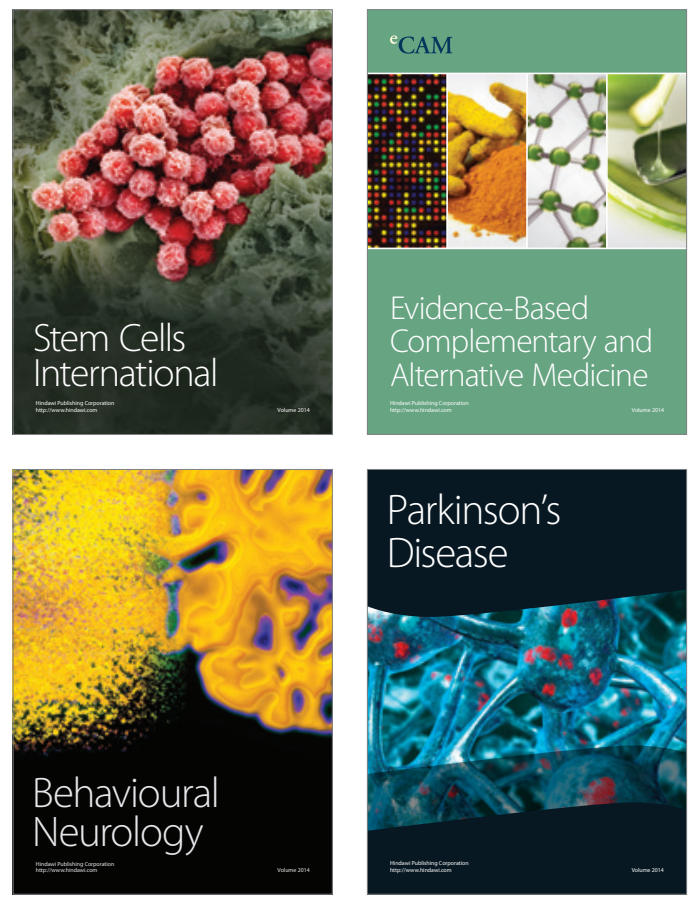
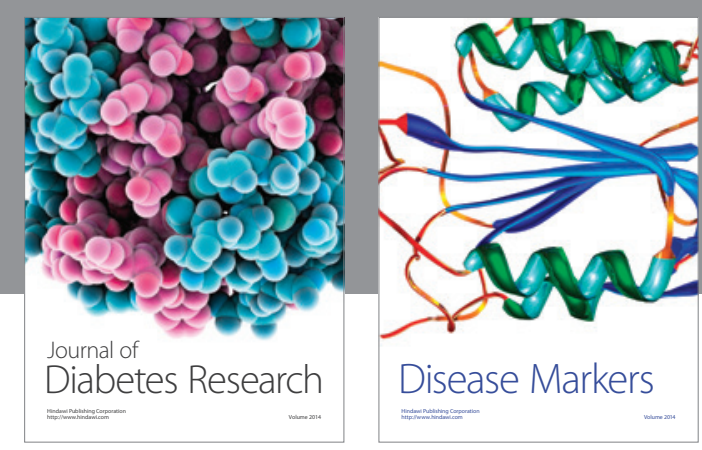

Disease Markers
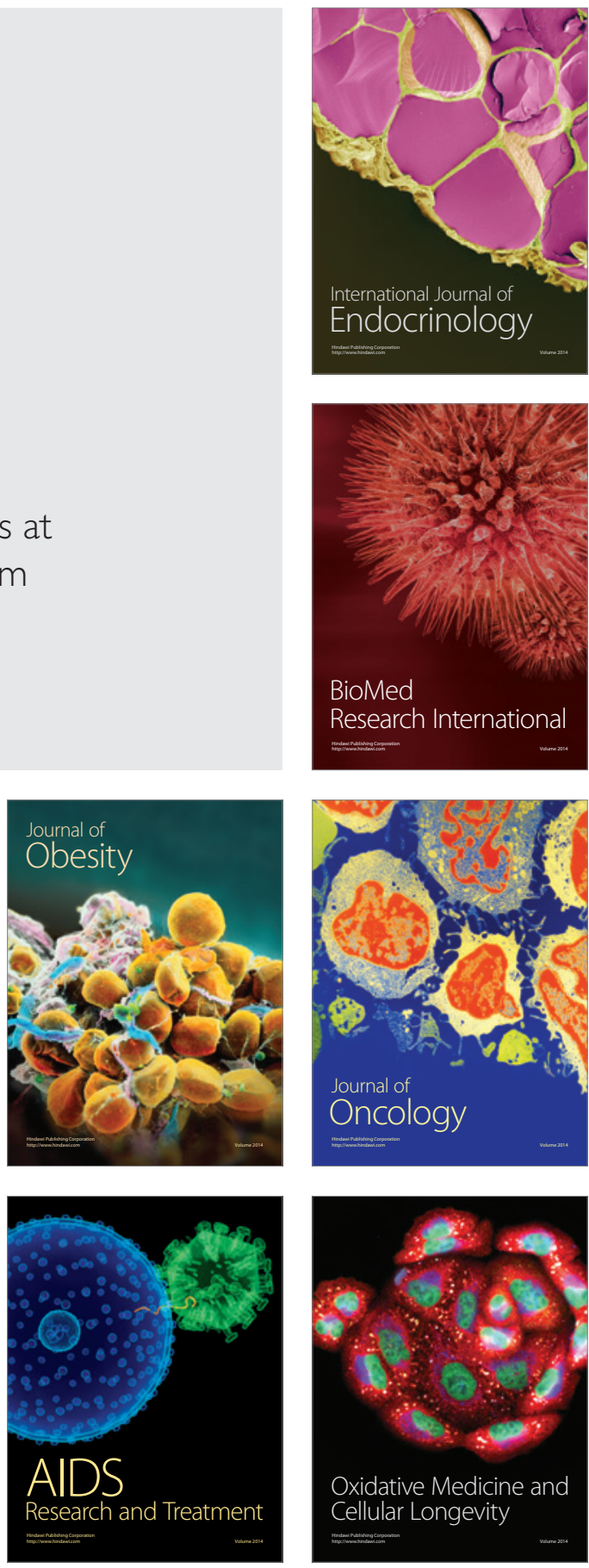